\title{
Asymptomatic Bacteremia in Children Infected with Human Immunodeficiency Virus in Calabar, South-South, Nigeria
}

\author{
Sunday O. Ochigbo*, Offiong E. Ikpeme, Atana U. Ewa and Ntia A. Happiness
}

Department of Paediatrics, University of Calabar, Calabar, Nigeria

\begin{abstract}
Background: Asymptomatic Human Immunodeficiency Virus (HIV) infected children are prone to serious bacterial infection as a result of poor immunity.

Objective: This was to determine the common pathogenic organisms responsible for bacteremia among asymptomatic HIV infected children attending the outpatient clinics at the University of Calabar Teaching Hospital, Nigeria.

Subjects and Methods: This prospective non randomized cross-sectional study was conducted over 6 months from January 1st to 30th June 2014 among cohorts of HIV infected Anti-retroviral (ARV) naive and experienced children attending the clinic. Ethical approval was obtained from the ethical committee of the institution. Blood culture specimens were collected from all HIV infected patients who met the criteria for enrollment. All specimens with isolated bacteria organisms were treated with antibiotics according to the sensitivity pattern, in addition to administered Anti-retroviral drugs.

Results: Out of the total of 109 patients recruited, 38(34.9\%) had bacteremia. There were more males (57.9\%), and most of them (63.2\%) had Highly Active Anti-Retroviral Therapy (HAART) for more than 24weeks. Bacteremia was inversely proportional to the CD4 count. The commonest organism isolated was unclassified Coliforms (47.3\%) followed by Staphyloccocus aureus (39.5\%).

Conclusions: HIV infected children are prone to bacteremia especially among those with low CD4 count. Coliform was the commonest organism isolated. We recommend that HIV infected children be routinely investigated for bacteremia.
\end{abstract}

Keywords: Organisms, ARV, gentamyicin, Blood culture, CD4.

\section{INTRODUCTION}

The World Health Organization (WHO) estimates that approximately 3.4 million children were living with HIV at the end of 2011 with $91 \%$ of them in SubSaharan Africa. Each year in Sub-Saharan Africa, 4.6 million children die before reaching the age of 5 years, and the vast majority of these deaths were not fully investigated [1]. Human Immunodeficiency Virus (HIV) and Acquired Immunodeficiency Syndrome (AIDS) are responsible for $4 \%$ of the causes of death among children under 5 years [2]. Bacterial infection was responsible for the immediate cause of death of up to $26 \%$ of patients with HIV infections [3]. HIV-infected children are at high risk for serious bacterial infections with encapsulated organisms [4] particularly during the early years of life in contrast to HIV-infected adults with predominant Opportunistic Infections (Ols). In Nigeria, bacteremia is an important cause of morbidity and mortality among HIV-infected children [2].

There is paucity of data on bacteremia in HIV positive children in Calabar, Nigeria. Therefore, this study was conducted to determine the common pathogenic organisms responsible and their sensitivity

*Address correspondence to this author at the Department of Paediatrics, University of Calabar, Calabar, Nigeria; Tel: +23 47063930682;

E-mail: ochigbosunny@gmail.com pattern. It is aimed to guide health care providers to initiate appropriate antibiotic treatment.

\section{SUBJECTS AND METHODS}

This prospective non randomised cross-sectional study was conducted from January $1^{\text {st }}$ to $30^{\text {th }}$ June 2014 at the Children Outpatient Clinics (CHOP) of the University of Calabar Teaching Hospital (UCTH), Calabar, Nigeria. The tertiary health care facility provided treatment and care at no cost to all HIV infected children less than 15years. An average of 3 new patients per week and total of 10-20 children attended the thrice weekly clinic. The children were treated with Highly Active Antiretroviral drugs (HAART) in accordance with the current Integrated National Guidelines for HIV Prevention, Treatment and Care (2014). These children were without fever or any other illnesses during their clinic visit. The CD4 count did not determine the inclusion or exclusion into the study. Excluded from the study were children on steroids, chemotherapy, those with skin sepsis, ear discharge. Also excluded were children on antibiotics except patients on routine prophylactic co-trimoxazole. The very ill patients excluded from the study were admitted into the paediatric ward of the hospital for treatment and care.

Ethical clearance was obtained from the Ethical Committee of the institution as well as signed consent 
from the parents or caregivers. Each patient that met the inclusion criteria was recruited. A filled proforma form was opened for each patient; information recorded included the age, sex, anthropometry, CD4 count etc. After explanation of procedure to the parents/caregivers, three mililitres $(3 \mathrm{mls})$ of blood was collected from each subject under aseptic condition and put into the culture bottle for aerobic blood culture. The samples were taken within 30 minutes to the medical laboratory department of the hospital for analysis by a certified laboratory scientist. Children with positive blood culture samples were treated for 7 days in accordance with the sensitivity results. The study was terminated if the patient requested for transfer to another centre, requested for discharge against medical advice, voluntarily withdrew consent after initial approval and death of patient during the study.

Data was analysed using Statistical Package for Social Students (SPSS) version 20. Continuous variables were summarized using means, median and ranges as appropriate. Proportions were compared using Chi-square test of significance. A probability ( $P$ value) of less than 0.05 was considered statistically significant.

\section{RESULTS}

Of the 109 children recruited into the study, $38(34.9 \%)$ had bacteremia. There were more males $57.9 \%$. The mean age was \pm 6.3 years $\left(X^{2}=0.421\right.$; Pvalue 0.516$)$. The mean weight and height were $19.1 \mathrm{~kg}$ (8.9-34.5; SD 6.66) and 107.2cm (73.5-137; SD 16.6) respectively. The mean BMI was 15.7 , below the 50th percentile. Twenty four 24(63.2\%) had HAART for more than 24 weeks (Table 1). Most of the parents of the children studied were in social classes II and III according to the classification by Oyedeji et al. [5]. The parents of $28(73.7 \%)$ and $10(26.3 \%)$ children were from the low and high socioeconomic classes respectively.

The relationship between CD4 count and bacteremia is shown in Table 2. Proportionately, the lower the CD4 count the higher the percentage of bacteremia except CD4 count $>1,500$ cells $/ \mathrm{mm}^{3}$.

The organisms isolated were unclassified Coliforms $47.3 \%$, Staphylococcus aureus $39.5 \%$, Streptococcus pyogenes $7.9 \%$, and Serratia species $5.3 \%$.

\section{DISCUSSION}

The prevalence rate of $34.9 \%$ obtained from this study was similar to $37.1 \%$ recorded by Imade et al. [6] in Benin Nigeria from the same geopolitical zone like Calabar. It was however higher than $21 \%$ reported by Kolo et al. [7] in Minna, North-Central Nigeria and $21.4 \%$ reported by workers in Uganda and Zimbabwe $[8,9]$. It is much higher than $12.8 \%$ reported by Berkley et al. [10] in Kenya. Our findings was corroborated by results of hemoculture antimicrobial susceptibility tests from HIV infected patients at Siriraj hospital, Bangkok, thailand. The percentages of positive hemocultures though slightly lower were $24.6 \%, 21.4 \%, 23.9 \%$, and $28.5 \%$ in 1996, 1997, 1998, and 1999, respectively [11]. The high prevalence observed could be attributed to the low socieconomic status of the parents of our subjects, ignorance and related poor personal hygiene. Hence the vicious cycle of HIV infection malnutrition and bacterial infection is maintained. On the other hand HIV awareness, availability of diagnostic facilities and ARVs encouraged the demand for services. Surprisingly, bacteremia was common among children

Table 1: Age, Sex and Duration of HAART among 38 HIV Positive Children with Bacteremia

\begin{tabular}{|c|c|c|c|c|c|c|c|}
\hline \multirow{2}{*}{ Characteristics } & \multicolumn{2}{|c|}{ Bacteremia } & \multicolumn{2}{|c|}{ No Bacteremia } & \multirow{2}{*}{ Risk ratio } & \multirow{2}{*}{$x^{2}$} & \multirow{2}{*}{$P$ value } \\
\hline & $\mathbf{N}$ & $\%$ & $\mathbf{N}$ & $\%$ & & & \\
\hline \multicolumn{8}{|l|}{ Age } \\
\hline $2-6 y r s$ & 17 & $(44.7)$ & 21 & $(55.3)$ & & & \\
\hline $6-12 y r s$ & 21 & $(55.3)$ & 17 & $(44.7)$ & 1.24 & 0.421 & 0.516 \\
\hline \multicolumn{8}{|l|}{ Sex } \\
\hline M & 22 & $(57.9)$ & 16 & $(42.1)$ & & & \\
\hline $\mathrm{F}$ & 16 & $(42.1)$ & 22 & $(57.9)$ & 0.73 & 0.947 & 0.330 \\
\hline \multicolumn{8}{|c|}{ Duration of HAART } \\
\hline$<24$ wks & 14 & $(36.8)$ & 24 & $(63.2)$ & & & \\
\hline$>24 w k s$ & 24 & $(63.2)$ & 14 & $(36.8)$ & 1.71 & 2.63 & 0.105 \\
\hline
\end{tabular}


aged 6-12 years. One would have expected a higher level of immunity among this age group. This observation could be due to the slow progression of the disease in some children identified as slow progressors or poor adherence to Anti-retroviral drugs.

Table 2: Relationship between Bacteria and CD4 Count in HIV Positive Samples

\begin{tabular}{|c|c|c|}
\hline $\begin{array}{c}\text { D4 Count Range } \\
\text { (cells/mm } \mathbf{3} \text { ) }\end{array}$ & No Examined & No Positive (\%) \\
\hline \hline$\geq 1500$ & 13 & $4(30.8 \%)$ \\
\hline$>750-1500$ & 30 & $7(23.3 \%)$ \\
\hline$>500-750$ & 38 & $12(31.6 \%)$ \\
\hline$>350-500$ & 18 & $9(50 \%)$ \\
$<350$ & 10 & $6(60 \%)$ \\
\hline Total & 109 & $38(34.9 \%)$ \\
\hline
\end{tabular}

Majority of the infected children had been on HAART for more than 24weeks. It is expected that after commencement of HAART for 24weeks and beyond there should be increased immunological response to more than $50 \%$ of baseline CD4 count, and viral load should be undetectable. Surprisingly, our study showed that children who received HAART for greater than 24 weeks were more infected with HIV. The reason for this is unclear; however drug adherence could not be entirely excluded as a possible reason for this observation. There are no facilities in our centre to measure the blood levels of the drugs to objectively determine the level of adherence. It is recommended that a study to measure blood levels of ARV drugs and bacteremia be conducted.

The inverse relationship between bacteremia and CD4 count could be due to improvement in the immunological status following the use HAART and corresponding decline of co-morbidities and other opportunistic infections. The commonest organism isolated from this study was Coliforms and closely followed by Staphylococcus aureus. In Benin Staphylococcus aureus was the commonest isolate in their study followed by Escherichia coli [6]. In Abuja, Obaro et al. [12] identified Staphylococcus aureus as the most common organism causing bacteremia. These findings were entirely at variance with the Watoto (ARROW) clinical trial in Uganda and Zimbabwe where Salmonella specie was the most common pathogen isolated, followed by Escherichia coli and Staphylococcus aureus [8] Coliforms are normal commensals of the digestive system while
Staphylococcus aureus are common on the skin. These are potential sources of acquistion of infection due to poor personal hygiene.

Most of the organisms were sensitive to Gentamycin which had been one of the first line drugs of our institutional antibiotic policy [13]. Gentamycin is a parenteral antibiotic, so its abuse is less compared to other oral antibiotics. However, in Benin Gentamiycin was the second most sensitive antibiotic after ceftriaxone.

\section{CONCLUSION}

This study showed that Coliforms and Staphylococcus aureus were organisms responsible for bacteremia in HIV-infected children in our centre. Children with low CD4 count were more predisposed to bacteremia. Clinicians therefore should have high index of suspicion in this group of children hence the need for regular blood culture. In addition, parents should be educated on nutritional therapy as an adjuvant to treatment in HIV infected children.

\section{LIMITATIONS}

Inability to perform anaerobic culture.

\section{COMPETING INTEREST}

Authors have no financial relationship with Presidents Emergency Plan for AIDS Relief (PEPFAR).

\section{ACKNOWLEDGEMENT}

We acknowledge the assistance of Mrs. Adie $\mathrm{N}$ Uduak, the Deputy Director Laboratory, University of Calabar Teaching Hospital, Calabar for her immense assistant to ensure analyses of the samples.

\section{REFERENCES}

[1] World Health Organization. Treatment of children living with HIV. $\quad$ www.who.int/hiv/topics/paediatric/hiv-paediatricinfopage.

[2] The State of the World Children, UNICEF 2011; pp. 88-91.

[3] James $\mathrm{A}$, Brett $\mathrm{S}$, Isaiah $\mathrm{M}$, et al. Bacteremia among children admitted to a rural hospital in Kenya. N Engl J Med 2005; 352: 39-47.

http://dx.doi.org/10.1056/NEJMoa040275

[4] Hatherill M. Sepsis predisposition in children with human immunodeficiency virus. Pediatr Crit Care Med 2005; 6: S928.

[5] Oyedeji GA. Socioeconomic and cultural background of hospitalized children in llesa. Nig J Paediatr 1985; 12: 111-7.

[6] Imade PE. Incidence of bacteremia in antiretroviral-naive HIV-positive children less than five years of age in Benin city, Nigeria. Libyan J Med 2010; 5: 4631-3. http://dx.doi.org/10.4176/090910 
[7] Kolo O, Galadima M, Daniyan S, Abalaka M, Talatu B. Bacteremia in Children infected with HIVIAIDS in Minna, Niger State, Nigeria. Br Micro Res J 2015; 9: 1-7. http://dx.doi.org/10.9734/BMRJ/2015/17110

[8] Mwambazi-Mweene, Mwate E. Effect of bacteraemia and HIV infection on treatment outcome in children with severe acute malnutrition admitted to the University Teaching Hospital Malnutrition Ward, Lusaka, Zambia.

[9] Musiime V, Cook A, Bakeera-Kitaka S, et al. Bacteremia, Causative Agents and Antimicrobial Susceptibility Among HIV-1-infected Children on Antiretroviral Therapy in Uganda and Zimbabwe. ARROW Trial Team.

[10] James A, Berkley, M, Brett SL, et al. Bacteremia among children admitted to a rural hospital in Kenya. Eng $\mathrm{J}$ Med 2005; 352: 39-47.

http://dx.doi.org/10.1056/NEJMoa040275
[11] Sirajaj S, Chokephaibulkit K, Yungyuen T, et al. Bacteremia and antimicrobial susceptibilities in HIV-infected patients at Siriraj Hospital. S East Asian J Trop Med Public Health 2005; 36: 347-51.

[12] Obaro S, Lawson L, Essen U, et al. Community acquired bacteremia in young children from central Nigeria-A pilot study. BMC Infect Dis 2011; 11: 137. DOI: 10.1186/14712334-11137

[13] Anah MU, Udo JJ, Ochigbo SO, Abia-Bassey LN. Neonatal septicaemia in Calabar, Nigeria. Trop Doct 2008; 2: 126-8. http://dx.doi.org/10.1258/td.2006.006037 